\title{
Fat Injection for Restoration of Facial Volume and Aesthetics Versus Conventional Facelift Surgery
}

\author{
AHMED F. EL-SHERIF, M.D.*; IBRAHIM H. KAMEL, M.D.* and \\ AMR ABD EL-MONIEM EL-NAGGARY, M.D.** \\ The Department of Plastic and Reconstructive Surgery, Faculty of Medicine, Ain Shams University* and \\ The Department of Plastic Surgery, Faculty of Medicine, Fayoum University**
}

\begin{abstract}
Facial rejuvenation has increased in demand recently. Standard facelift surgery has been challenged by more recent, less invasive techniques; those are heralded to provide a safer and cheaper alternative to selected patients. In this work, we compare simple fat grafting to a full facelift procedure. Patient satisfaction scores were statistically significantly higher in the fat graft group, while surgeons' satisfaction was comparable in both groups. Complication rates were higher in the full facelift group. Blepharoplasty combined with lipofilling seems to be an effective and satisfactory means for facial rejuvenation in suited female patients.
\end{abstract}

Key Words: Facelift - Fat injection - Rhytidectomy - Autologous fat transfer.

\section{INTRODUCTION}

Facelift surgery (rhytidectomy) has long been the gold standard for treating the aging face in both sexes [1]. The procedure is a tedious one, requiring surgeon experience, patient understanding, and acknowledgment of the risks that such a procedure may carry from both parties $[2,3]$.

To understand the corrective procedure, surgeons have to be aware of the facial anatomy as well as the anticipated pitfalls and potential complications [2]. Many authors have attempted to provide their explanations for the physiology of facial aging [4]; the current consensus comes to conclude that facial aging is the outcome of three main tissue changes: (1) Bony changes with loss of support and remodeling, (2) Soft tissue changes (facial muscles, facial fat and retaining ligaments "sagging"), and (3) Skin changes.

Consequently, an increasing number of authors do not support the idea of one single procedure as the standard solution for the management of facial aging. Instead, tailoring the procedure to the patient's unique parameters and desires is advocated [5]. Many surgeons, thus, are open to complementing the surgical aspect of the procedure with other additives such as chemical peeling, laser resurfacing, radiofrequency emitting devices (Ultherapy/ HIFU), thread lifting, botulinum toxin type A injection and/or platelet rich plasma meso-therapy injections [6-9].

In fact, with a sizable complication rate and a major surgical expertise prerequisite [10], many practitioners-and patients-would opt for less invasive and simpler options for facelift. Different areas of the face do have different impacts on the necessary procedure appropriate for correcting the aging part of the face [11], and thus the customization of the procedure used can be done according to the aging area involved.

Fat grafting (lipofilling) has gained popularity in the recent years for both therapeutic and aesthetic purposes [12]. Facial fat augmentation is a simple, relatively inexpensive, safe, and quick procedure for facial rejuvenation that has garnered the interest of both surgeons and patients and has been reported to achieve satisfactory outcomes in the literature $[13,14]$. The procedure can be performed in an outpatient setting and the recovery is virtually instantaneous, versus the tiresome journey to recovery in standard facelift surgery. However, it is sometimes regarded inferior to standard facelift surgery, which renders some surgeons and patients reluctant to adopt the procedure [1].

In this work, we attempted to compare simple fat grafting versus full facelift surgery for facial rejuvenation. 


\section{MATERIAL AND METHODS}

Thirty female patients seeking facial rejuvenation intervention for aging changes were divided into two equal groups, both groups were age matched. All cases had blepharoplasty performed (done through a trans-conjunctival or a sub-ciliary approach), together with fat injection (Group A), or as part of a full facelift surgery (Group B).

In Group A, a trans-conjunctival or subciliary blepharoplasty was done according to the age group and necessity of each individual case, together with facial fat injection over fixed bony prominent areas and other folds, as the nasolabial and mentolabial folds. Fat was harvested either from the abdominal area or the inner thighs. A 500mL Ringer's Lactate solution was always used, to which $1 \mathrm{mg}$ of epinephrine and $5 \mathrm{~mL}$ of Lidocaine $2 \%$ were added. The average amount of aspirated fat for facial injection was usually around $300 \mathrm{~mL}$. The total amount aspirated was then centrifuged at $4000 \mathrm{rpm}$ for 10 minutes, then the residual oily layer at the top was disposed of and the fat was centrifuged again for another 5 minutes at a lower speed of $2500 \mathrm{rpm}$. The average amount of $300 \mathrm{~mL}$ aspirated fat would finally resolve to around $200 \mathrm{~mL}$, after the serum and oil were disposed of.

Certain injection points were fixed for all cases, and additional points were included when necessary. The fixed points were the cheek area over the zygomatic arch, the nasolabial folds, the mentolabial folds, and the angle of the mandible and jowl area.

In Group B, the patients underwent blepharoplasty as well as a facelift surgical procedure with Superficial Musculoaponeurotic System (SMAS) plication. This included dissection in the plane superior to the SMAS to expose it followed by its redraping to the deeper immobile parotidomasseteric fascia and trimming of any excess SMAS. The patients adhered to complete bed rest for 24 hours post-operatively, followed by head elevation for the week that followed. Post-operative painkillers were prescribed together with ice packs.

The results were compared through photographic comparison of pre and post-operative results in both groups. The evaluation was done by the patients themselves, and by two other independent surgeons, and a total score out of 3 points was given for each case individually by each evaluator (1: Not satisfied, 2: Moderately satisfied, and 3: Extremely satisfied).
Quantitative data were tabulated and analyzed using SPSS v. 20 (IBM, United States).

\section{RESULTS}

The mean age of the patients was 44.5 years (range: $40-72$ years) for Group A and 47.8 years (range: 42-69 years) for Group B. Figs. $(1,2)$ show pre-operative and post-operative (at week 2 ) images of patients from Group A, while Figs. $(3,4)$ show pre-operative and post-operative images (at week 4) if patients from Group B). Group B's final images were obtained at later follow-up visits to allow for complete resolution of postoperative edema and a fair judgement on final outcome.

The rating for satisfaction to the surgical outcome was evaluated through comparing pre and post-operative photos by the patients themselves and by two other surgeons as mentioned before. In Group A, 9 patients were extremely satisfied with the outcome (3 points) and did not seek any further correction. Four patients were moderately satisfied ( 2 points) and all sought another fat injection procedure to restore more facial volume. One patient scored 1 point, but still opted for another fat injection surgery preferring it to facelift. Only one patient requested a facelift procedure, also evaluating the outcome as a single point. The total score for Group A patients' satisfaction was 37 points. The mean of the two surgeons' evaluation for the cases in Group A was 33 points.

In Group B, the patients self-evaluated the surgical outcome as follows: Three patients were extremely satisfied ( 3 points), six were moderately satisfied ( 2 points), and six were not satisfied scoring a single point. The total score for patients' satisfaction in Group B was 27 points. The average surgeons' satisfaction score was 32 points.

Reasons provided by the patients for dissatisfaction in Group B included visible scars $(n=8$, $53.3 \%)$, pixie ear deformity $(n=3,20 \%)$, the need of extra skin lift $(n=2,13.3 \%)$, and inadequate facial volume restoration $(\mathrm{n}=2,13.3 \%)$.

Scores comparison revealed there was a statistically significant difference in favor of the fat grafting technique when it came to patient satisfaction $(p<0.001)$ while this difference was not statistically significant when it came to the surgeons' opinion $(p=0.472)$. 

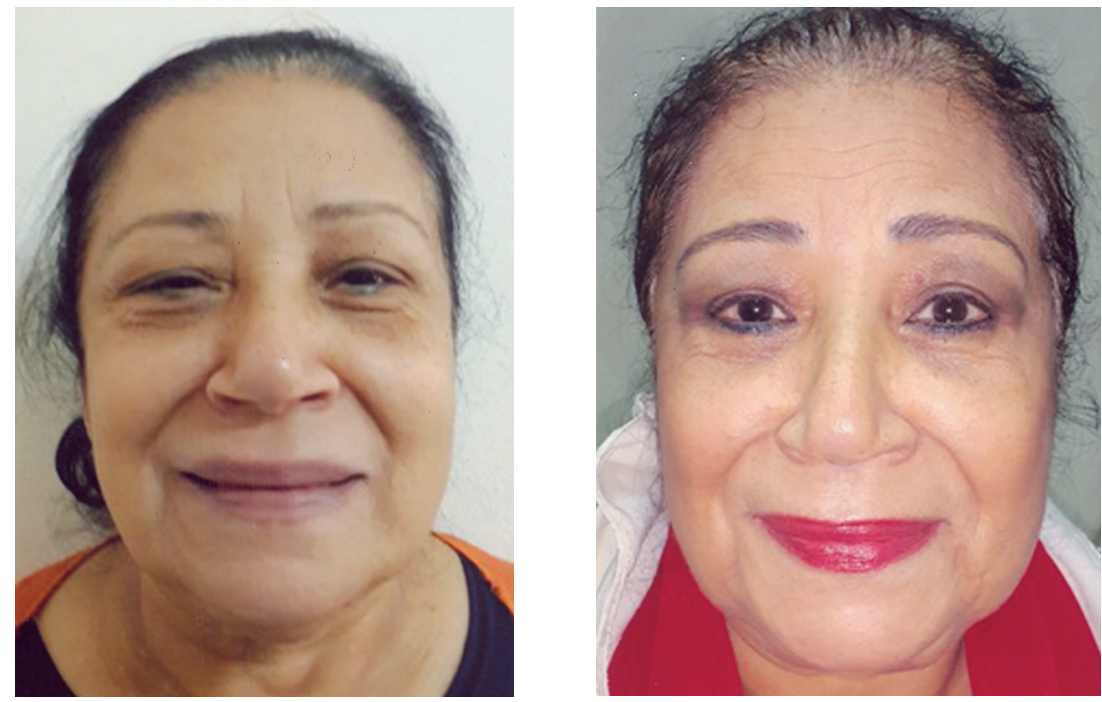

Fig. (1): Pre-operative (left panel) and postoperative (right panel) images of a sample patient (52 years old) from Group A.
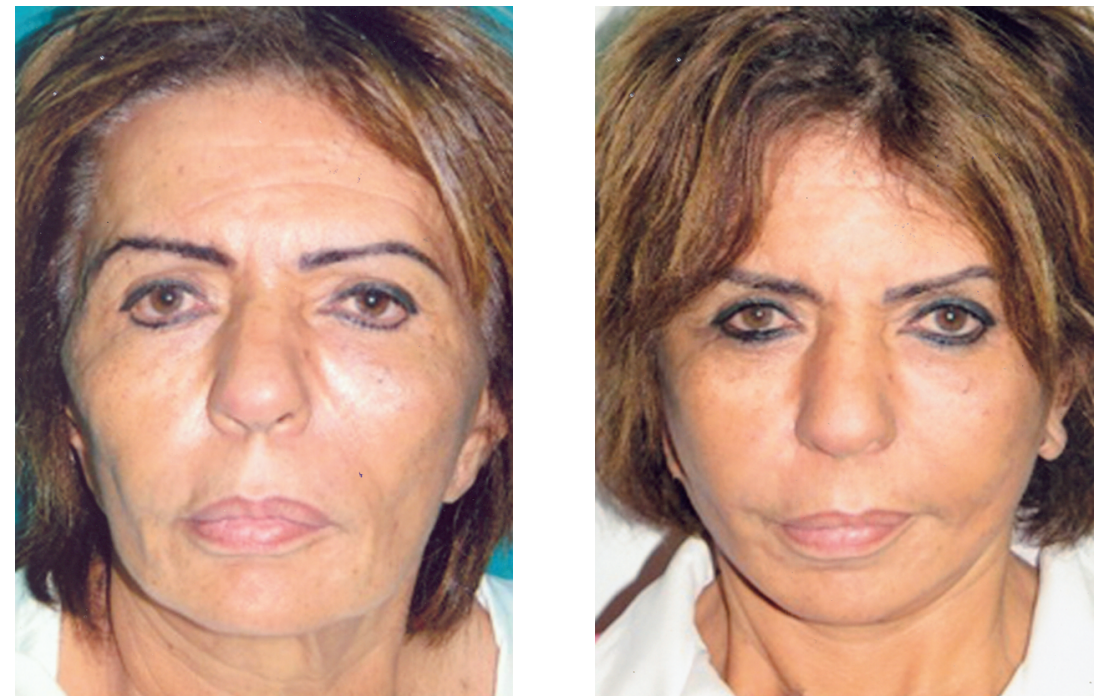

Fig. (2): Pre-operative (left panel) and postoperative (right panel) images of a sample patient (48 years old) from Group A.
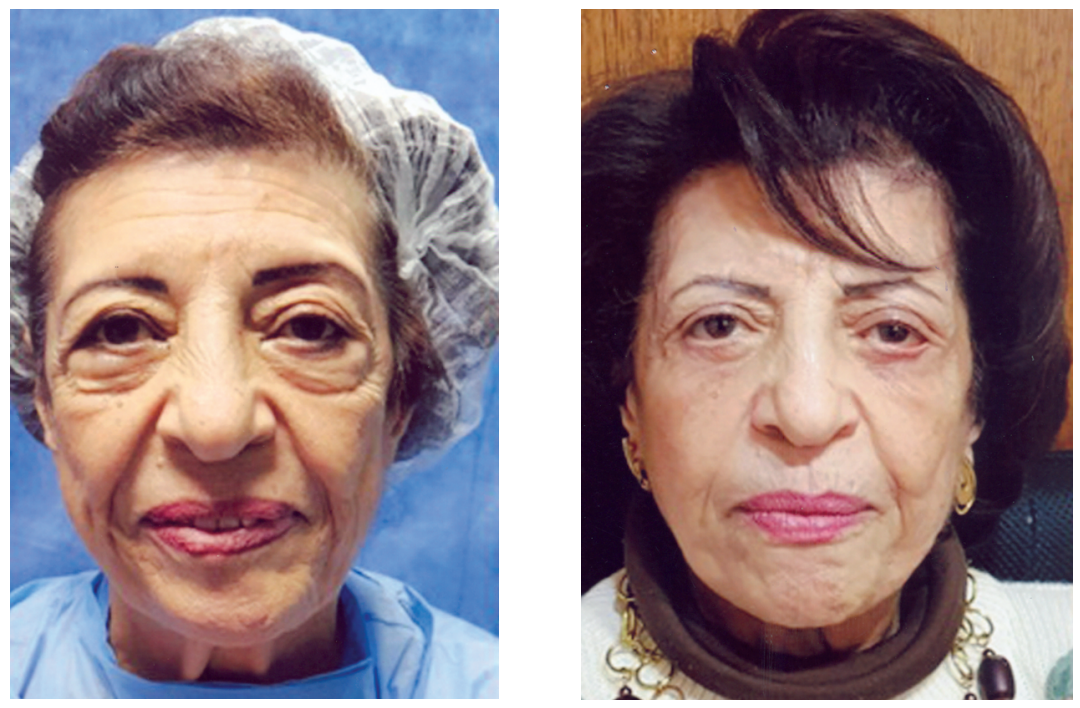

Fig. (3): Pre-operative (left panel) and postoperative (right panel) images of a sample patient (57 years old) from Group B.. 

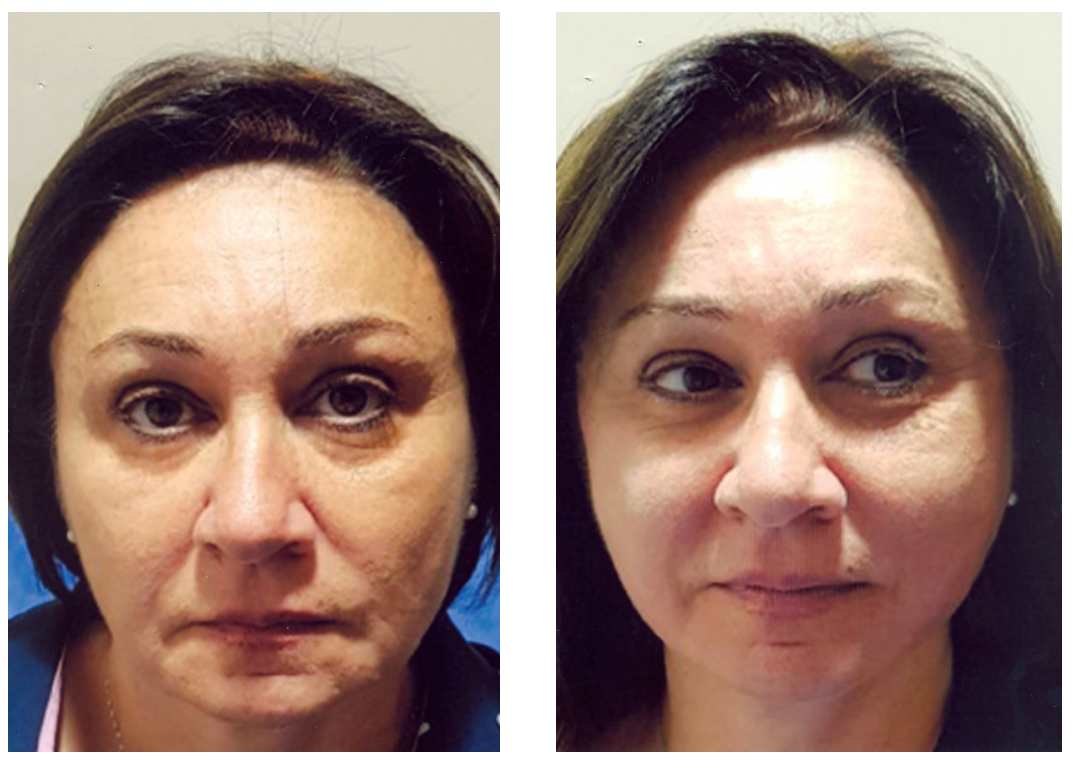

Fig. (4): Pre-operative (left panel) and postoperative (right panel) images of a sample patient (48 years old) from Group B.

\section{DISCUSSION}

In this work, we compared simple fat grafting with blepharoplasty to a full surgical facelift procedure for facial rejuvenation in females with aging faces. Our results showed comparable outcome in both groups, with the fat grafting group exhibiting higher degrees of overall satisfaction scores with the procedure. To the beast of our knowledge, this is the first study to pit fat grafting against surgical face-lifting using quantitative measures.

Traditional surgical facelift procedures are losing their established place as the gold standard to treat any form of facial sagging. This may be owed to a higher incidence of complications, the tedious time during surgery and prolonged postoperative recovery period [1]. In his analysis of 93 facial rejuvenation patients [15], Swanson has demonstrated that non-surgical procedures such as thread lifting or laser resurfacing, as well as various surgical procedures such as blepharoplasty, fat injection, and forehead lift, along with facelift, all significantly contributed to the final outcome and patient satisfaction. Subdivision analysis was not, however, carried out to demonstrate superiority of one adjunct technique over the other.

In their article [16], Stein et al., presented their theoretical work of a beautiful midface by combining the shaping of the mid-face fat with each lower eyelid blepharoplasty to achieve a "Phi point". This agrees with our work, that blepharoplasty and facial fat reshaping will achieve aesthetic results without the need of full facelift.
Donofrio [17] has argued for restoring face contour and replacing volume by fat injection. The author emphasized the strong utility of fat injection as an adequate procedure to restore facial aesthetics together with decreasing the post-operative down time.

In another recently published work [18], Minimal Undermining Suspension Technique (MUST), combined eyebrow and mid-face lift via temporal access while trying to avoid the hazards of full facelift, was demonstrated to be an effective and safe means for facial rejuvenation. Complications were limited to only ecchymosis of the orbiculotemporal region and a cheek dimple due to the suspension suture in only 2 cases. According to the MERZ Aesthetic Scales, this technique showed significant improvement at 12 months post-operatively. This cements the idea that other less invasive techniques may provide results just as superior as a full facelift, while avoiding the hazards of the operative complications.

In conclusion, minimal invasive surgery provides a useful and effective alternative for facial rejuvenation. Blepharoplasty and fat injection achieved comparable results to full facelifts while perceived as statistically significantly more satisfactory by the patients.

\section{REFERENCES}

1- Carniol P.J. and Ganc D.T.: Is there an ideal facelift procedure? Curr. Opin. Otolaryngol. Head Neck Surg., Aug., 15 (4): 244-52, 2007.

2- Pourdanesh F., Esmaeelinejad M., Jafari S.M. and Nematollahi Z. Facelift: Current Concepts, Techniques, and 
Principles. In: A Textbook of Advanced Oral and Maxillofacial Surgery Volume 3. In Tech., p. 653-79, 2016.

3- Sarcu D. and Adamson P.: Psychology of the Facelift Patient. Facial Plast. Surg., Jun., 33 (3): 252-9, 2017.

4- Sveikata K., Balciuniene I. and Tutkuviene J.: Factors influencing face aging. Literature review. Stomatologija, 13 (4): 113-6, 2011.

5- Wang R., Yang J., Guo K., Zhong A., Tong J., Xiong L., et al.: Asian Facelift Technique Refinement With High Patient Satisfaction: FACE-Q Report. Ann. Plast. Surg., May, Preprint 2018.

6- Zalesak B.: Facelift-current concept of complex facial rejuvenation. Cas Lek Cesk, 157 (6): 293-7, 2018.

7- Gonser P., Kaestner S., Jaminet P. and Kaye K.: Histological case-control study of peeling-induced skin changes by different peeling agents in surgically subcutaneous undermined skin flaps in facelift patients.. J Plast. Reconstr. Aesthet. Surg., Nov., 70 (11): 1660-5, 2017.

8- Taghizadeh F., Leibowitz A., Ramirez P. and Santos D.: Combining facelift with SmartLipo 1064-nm system, technique overview and retrospective analysis of 56 patients. J. Cosmet. Laser Ther., Apr., 17 (2): 80-5, 2015.

9- Kalra R.: Use of barbed threads in facial rejuvenation. Indian J. Plast. Surg. Off Publ Assoc. Plast. Surg. India. Oct., 41 (Suppl): S93-100, 2008.

10- Jacono A.A., Sean Alemi A. and Russell J.L.: A MetaAnalysis of Complication Rates Among Different SMAS Facelift Techniques. Aesthetic Surg. J., Feb., 2019.
11- Sulamanidze M., Sulamanidze G. and Sulamanidze C.: Elimination of Aesthetic Deformations of the Midface Area Our Experience. Aesthetic Plast. Surg., Jun., 42 (3): 774-90, 2018

12- Denadai R., Buzzo C.L., Raposo-Amaral C.A. and RaposoAmaral C.E.: Facial Contour Symmetry Outcomes after Site-Specific Facial Fat Compartment Augmentation with Fat Grafting in Facial Deformities. Plast. Reconstr. Surg., Feb., 143 (2): 544-56, 2019.

13- Sezgin B. and Ozmen S.: Fat grafting to the face with adjunctive microneedling: A simple technique with high patient satisfaction. Turkish J. Med. Sci., Jun., 48 (3): 592-601, 2018.

14- Rohrich R.J. and Afrooz P.N.: Finesse in Face Lifting: The Role of Facial Fat Compartment Augmentation in Facial Rejuvenation. Plast. Reconstr. Surg., Jan., 143 (1): 98-101, 2019.

15- Swanson E.: Outcome analysis in 93 facial rejuvenation patients treated with a deep-plane face lift. Plast. Reconstr. Surg., Feb., 127 (2): 823-34, 2011.

16- Stein R., Holds J.B., Wulc A.E., Swift A. and Hartstein M.E.: Phi, Fat, and the Mathematics of a Beautiful Midface. Ophthal Plast. Reconstr. Surg., 34 (5): 491-6, 2018.

17- Donofrio L.M.: Panfacial Volume Restoration with Fat. Dermatologic Surg., Nov. 1, 31 (s4): 1496-505, 2005.

18- Gualdi A., Cambiaso-Daniel J., Gatti J., Bertossi D., Pietramaggiori G., Scherer S.S., et al.: Minimal Undermining Suspension Technique (MUST): Combined Eyebrow and Mid-face Lift via Temporal Access. Aesthetic Plast. Surg., Feb., 41 (1): 40-6, 2017. 